\title{
Modelos descriptivos de corona para Laurel (Cordia alliodora) bajo sistemas agroforestales en el Bosque Protector Sumaco, Ecuador
}

\author{
Álvaro Cañadas ${ }^{1 *}$, Mykola Korol $^{2}$, Carlos Zambrano $^{3}$ y Bolívar Camacho ${ }^{4}$ \\ ${ }^{1}$ Universidad Técnica del Norte, Facultad de Ingeniería en Ciencias Agropecuarias y \\ Ambientales, Av. 17 de Julio 5-21 y José Córdova, Ibarra, Ecuador. Teléfono: 00593-6-2997800. \\ ${ }^{2}$ Universidad Técnica Estatal Forestal de Ucrania, Tschuprynkastr; 103, 79044 Lviv, Ukraine. \\ ${ }^{3}$ Universidad Técnica Estatal de Quevedo, Km 1 1/2 vía $S^{\text {to }}$ Domingo, Ecuador. \\ ${ }^{4}$ Universidad Técnica de Manabí, Av. Urbina y Che Guevara, Portoviejo, Ecuador \\ *Autor principal/Corresponding author,email: alvarogustavo.canadaslopez@alumni.uni-goettingen.de
}

Editado por/Edited by: D. F. Cisneros-Heredia, Ph.D.(c)

Recibido/Received: 10/01/2012. Aceptado/Accepted: 10/15/2012.

Publicado en línea/Published on Web: 12/28/2012. Impreso/Printed: 12/28/2012.

\begin{abstract}
The ability to describe the shape and size of a tree crown is an important pre-requisite for the estimation of the stability of standing forest trees. The paper describes a crown model for laurel in agro forestry systems of the Protected Forest Sumaco. The empirical data base includes altogether 207 Laurel sample trees. A stem analysis was carried out for each sample tree crown ratio, crown length, crown surface area and crow projection. The sample trees were grown in 3 agro forestry stands which had been subjected to different thinning regimes. To determine the crown width a lineal relationship existed between DBH and crown width. The modification of the models in direction toward a non-lineal relationship not showed improvements in the estimate of this parameter. The crown base, the model proposed by Durský (2000) was demonstrated the best $\mathrm{r}^{2}(0.84)$. The distortion value of this model was closed to the ideal value of $0(0.0083)$ and mean squared error was $1.49 \mathrm{~m}$. This model was adjusted to the characteristics of Laurel. It was developed a model in order to determinate the crown length. The model presented an $\mathrm{r}^{2}$ of 0.71 . This means that, $71 \%$ of total variance observed in Laurel can be explained for this parameter. The developed models of this research could be used to establish and calculate other forest attributes of Laurel.
\end{abstract}

Keywords. crown base, crown model, crown ratio, crown length, crow projection

\section{Resumen}

La habilidad de describir la forma y tamaño de la corona de los árboles es un importante pre-requisito para la estimación de la estabilidad de los árboles de un bosque. Este artículo describe modelos de corona para el Laurel (Cordia alliodora), adaptadas a las condiciones de crecimiento de sistemas agroforestales. La base de datos empíricos incluye un total de 207 observaciones. Un análisis de corona basado en el largo, ancho, base de corona, largo, radio y proyección de corona. Los árboles muestreados han crecido en sistemas agroforestales de 10 años de edad, los cuales fueron raleados a diferentes intensidades. Para determinar el ancho de corona existió una relación lineal entre DAP y ancho de corona. La modificación de los modelos en dirección hacia una relación no lineal no muestra mayores mejoramientos en la estimación de este parámetro. La base de la Corona, el modelo desarrollado por Ďurský (2000) es el que mayor $\mathrm{r}^{2}(0.84)$ presentó, los valores de distorsión del mencionado modelo se acercan a los valores ideales de $0(0.0083)$ y el cuadrado medio del error fue de 1.49 metros. Este modelo se ajustó a las características del Laurel. Se desarrolló un modelo para establecer el largo de la corona, el cual presentó un $\mathrm{r}^{2}$ de 0,71 . Esto significa que únicamente el $71 \%$ de la variación observada en el Laurel para este parámetro puede ser explicada. Los modelos desarrollados dentro de esta investigación pueden ser utilizados para comprobar y calcular otro tipo de atributos forestales del Laurel.

Palabras Clave. ancho de la corona, base de corona, largo de la corona, radio de la corona, proyección de corona 


\section{Introducción}

De acuerdo al Ministerio del Ambiente del Ecuador, MAE [1] la superficie de bosques nativos en el Ecuador, abarca aproximadamente 8.8 millones de hectáreas. De estas, los manglares ocupan 227,300.00 has. Por su difícil accesibilidad, en la actualidad únicamente $600 \mathrm{mil}$ has de bosques nativos presentan condiciones económicas para ser manejados silviculturalmente, mientras que las tierras con potencial para ser forestadas alcanzan una extensión de más de 2 millones de hectáreas. Las plantaciones forestales tan sólo representan un total de 160,000.00 has [2]. De ahí que, se cree que en países tropicales el recurso bosque es abundante y que esta esperando ser explotado, de manera que la atención en el Ecuador se ha centralizado más sobre la explotación y utilización antes que sobre su plantación y crecimiento. Desde este último punto de vista, la tendencia en nuestro país ha sido considerar a la plantación de árboles como un arte, sin prestar atención a los costos involucrados y sin preocuparse aún en cual va a ser el futuro preciso de su utilización [3]. La reflexión sobre este argumento radica en que los productos forestales, a diferencia de otras materias primas, nunca se planifican para satisfacer las necesidades de una industria cada vez más creciente, sino simplemente se establecen plantaciones amparadas en el supuesto de que de algún modo estas van a ser explotadas, aprovechadas y utilizadas [4, 5].

Los forestales necesitan tener información útil sobre diversos parámetros concernientes a árboles y rodales para mejorar la planificación forestal con la finalidad de tomar decisiones sobre un manejo silvicultural apropiado [6]. La modelación forestal suministra información adicional para la planificación y estimación de producciones. El modelaje forestal representa el promedio de cómo los árboles crecen y como la estructura del bosque son modificados. De ahí que, estos se constituyen en una herramienta flexible para predecir, con lo cual se puede enunciar una mejor formulación de políticas $[7,8]$. Los modelos de crecimiento forestal son entonces una herramienta útil para comparar alternativas de tratamiento. Dependiendo del nivel de detalle, de la disposición de datos y del grado de precisión requerida en la estimación, se dividen a los modelos forestales dentro de la siguiente tipología $[7,8]$ :

- Modelos de predicción regional y crecimiento de rodales: Producen información agregada sobre el desarrollo de la población de árboles dentro de un determinado juego de condiciones ambientales y una modificación dada de los atributos de un rodal por interferencia humana u otro tipo de perturbaciones.

- Modelos de clase de tamaño: Proyectan el desarrollo de un limitado número de árboles que representa la población total, cada árbol representativo tiene los atributos de una sub-población de árboles que son similares de una u otra manera.
- Modelos de árbol: Tratan el detalle de la morfología de coronas, ramas, forma de fuste y crecimiento de la raíz.

Modelos simples de árbol incluyen el incremento de diámetro-altura, mortalidad y sub-modelos como modelos de corona, los cuales describen la forma de la corona, como también el desarrollo de las mismas. El objetivo de tales modelos es predecir el desarrollo de un simple árbol dentro de un determinado rodal [9]. Por otro lado, la condición básica para el crecimiento arbóreo es la asimilación de $\mathrm{CO}_{2}$ que tiene lugar en la copa y que está ligada con la transpiración de manera directa. A causa de dicha asimilación se mantiene el flujo de savia vertical que garantiza que el agua y los nutrientes lleguen a las hojas y por lo tanto, representa una condición esencial para la fotosíntesis [10]. La distribución de los órganos de asimilación en las ramas y ramillas de copa, sirve a su vez para garantizar la obtención de la radiación necesaria para la fotosíntesis [11]. Pero la copa de los árboles es, además, un "tesoro de la diversidad" sin investigar. Por ejemplo, en las copas de los árboles tropicales se han descubierto en los últimos años más especies nuevas de insectos que en cualquier otro hábitat de la Tierra [12].

La copa de los árboles no es una estructura estática, sino que cada año se modifica con la formación de nuevos brotes, ramillas y ramas. Este cambio ocasiona una nueva distribución espacial de las hojas o acículas. Una de las causas que motiva este cambio es la competencia de copas dentro de un rodal $[13,14]$. Los requerimientos de espacio de las raíces y de la copa, que se incrementan con la edad, solo se pueden satisfacer si una parte de la población desaparece, reduciendo temporalmente los efectos de la competencia. Esta competencia, por la cual los individuos de los estratos inferiores ven impedido su desarrollo, se puede apreciar en las copas de los árboles. La forma de la copa y en especial su cobertura permite sacar conclusiones sobre las condiciones de crecimiento, la silvicultura y la competencia a la que ha estado sometido un árbol en concreto [15]. El estudio fue conducido en la provincia de Napo, parroquia de Wamaní, coordenadas UTM 9920000 y 2150000, a una elevación de $1.160 \mathrm{~m}$ sobre el nivel del mar con una temperatura promedio de $21^{\circ} \mathrm{C}$ (Figura 1).

La corona de un árbol tiene diversas funciones, no solamente para el árbol en si, sino que también para el medio ambiente en general. La investigación sobre la forma y desarrollo de la corona forestal es de actualidad [16]. La asimilación del carbono y la respiración son procesos en los cuales a través de la oxidación, una parte de la energía es liberada, dependiendo claro esta de la especie forestal y el tamaño de la corona de los árboles. Por tal motivo, la modelación de corona a menudo es utilizada como una variable de prognosis para determinar la evaluación del crecimiento de los árboles [7]. Por otro lado, la forma de la corona de los árboles es muy 


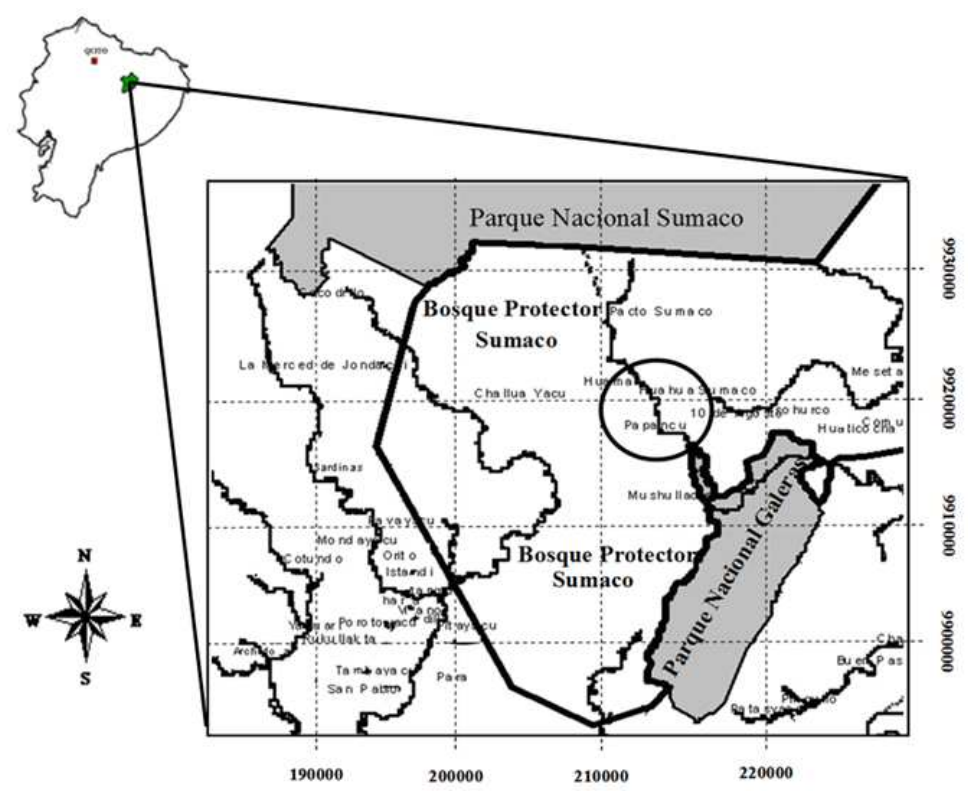

Figura 1: Ubicación geográfica del área de investigación agroforestal, parroquia Wamaní, provincia de Napo.

diversa y está influenciada por varios factores como la información genética, la edad, disponibilidad de espacio relacionadamente con la intensidad, forma de los raleos y las condiciones de sitio. Una influencia definitiva sobre el desarrollo de la corona y con esto la estabilidad del rodal contra el viento tiene la forma de manejar a un bosque [17].

La dimensión de la corona de los árboles tiene adicionalmente un gran significado para investigaciones ecológicas y económicas del crecimiento forestal, dado que, con su biomasa y área foliar actúa como el promotor primario de crecimiento arbóreo [18]. En el Ecuador existe muy pocos estudios sobre la descripción y el desarrollo de la corona en sistemas agroforestales provenientes del manejo de la regeneración natural en la Región Amazónica Ecuatoriana del Laurel (Cordia alliodora) [3]. La presente investigación tiene como objetivo probar diversos modelos sobre el ancho de corona, base de corona, largo de corona adaptadas a las condiciones de crecimiento de Cordia alliodora en sistemas agroforestales, en las comunidades de Wamaní, Wawa Sumaco y 10 de Agosto, Bosque Protector Sumaco.

\section{Métodos}

\section{Área de Estudio}

La estación pluviométrica representativa para el área de estudio es la estación de Challua Yacu, la cual registra una precipitación media anual de $4.000 \mathrm{~mm} / \mathrm{año} \mathrm{y} \mathrm{su}$ distribución durante el año es representada en la Figura 2 [19].

En la región del Tena en el mes de diciembre se establece un sistema predominante de vientos Alisios, los mismos que por efecto de la rotación terrestre son desviados en dirección NE. Estos vientos se manifiestan en

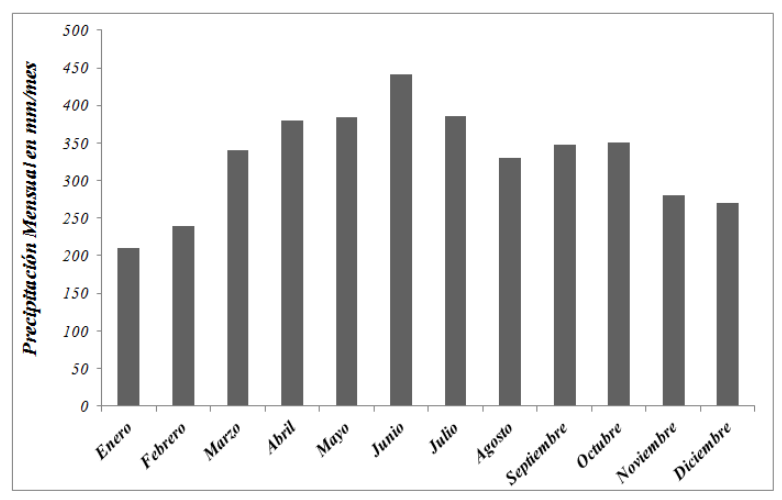

Figura 2: Distribución de la precipitación mensual en el área de Chalua Yacu.

forma de fuertes vientos provocando la disminución de vapor de agua y por ende la baja de la pluviosidad en la región. En los meses de abril y marzo, donde la corriente de El Niño (Calido y Húmedo) se encuentra en su apogeo, se suma a este, la presencia en la región amazónica de los vientos Alisios monzónicos en dirección NO cargados de vapor de agua, lo cual da como resultado el primer pico de lluvias en la región. Entre junio y julio mientras todo el Ecuador se encuentra un periodo seco, en la Cordillera Oriental se presenta el período de máxima lluvia. La causa de este fenómeno es la presencia de los vientos Alisios en dirección SE, los cuales están relacionados con una corriente fría proveniente del hemisferio sur. Por otro lado, cuando la Tierra va hacia el hemisferio sur después del equinoccio (octubre), la región oriental se ve influenciada por las corrientes de los vientos Alisios en dirección NO y la desviación de los mismos en dirección NE del hemisferio norte, provocando en la región el segundo pico de lluvias [20].

Los suelos de la comunidad Wamaní, Wawa Sumaco y 10 de Agosto pertenecen al gran grupo de suelos de- 


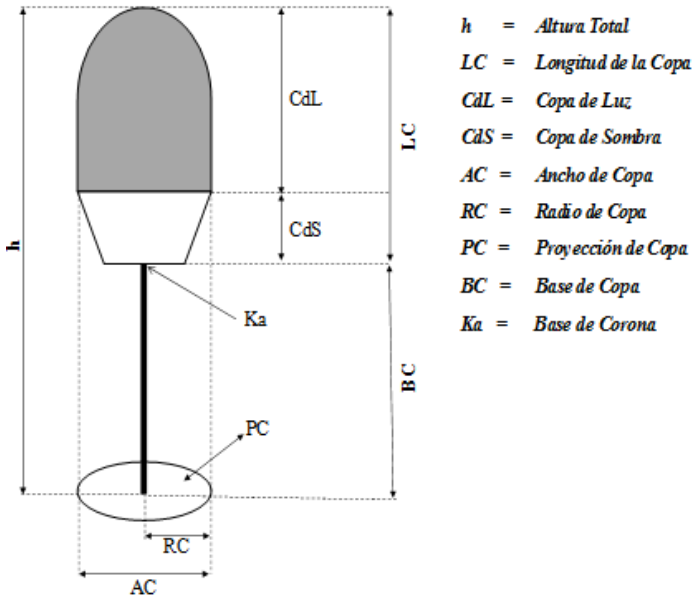

Figura 3: Representación esquemática del perfil de corona de un árbol.

nominados Hydrandepts. Estos suelos son generalmente negros en su horizonte superficial y pardo amarillo en los horizontes subsiguientes. Su capacidad de retención de humedad es mayor a la capacidad de campo. Por esta razón, los procesos de lixiviación (lavado) son continuos y la meteorización de los minerales primarios es casi completa, liberando cationes de $\mathrm{Al}^{+}$y cationes $\mathrm{H}^{+}$, los cuales contribuyen a la acidificación del suelo. Por otro lado, la acidez del suelo va disminuyendo conforme se aumenta la profundidad del perfil del suelo, esto se debe principalmente a la poca actividad radicular de la vegetación en esta área [21]. La zona de estudio pertenece a la zona de vida de Bosque Húmedo Tropical [22].

\section{Métodos de campo}

La Figura 3 muestra un modelo simplificado de una copa de un árbol con algunos de los parámetros característicos utilizados en la práctica forestal para estimar el crecimiento de la biomasa o para juzgar la estabilidad de un rodal. Para una descripción cuantitativa de la corona de Laurel bajo sistemas agroforestales en esta investigación, diferentes mediciones fueron obtenidas de acuerdo a la Figura 3 (i.e., largo de la corona, ancho de la corona, diámetro a la altura del pecho, altura comercial y altura total).

Para la determinación de los parámetros de la función de ancho de corona y base de corona se utilizó un Hipsómetro AGA, Esc 1:20, un total de 207 árboles de tres sistemas agroforestales de Wamaní (458 árboles/Ha., 10.7 años de edad), Wawa Sumaco (375 árboles/Ha., 9 años de edad) y 10 de Agosto (325 árboles/Ha., 8.5 años de edad) fueron medidos. Las características generales de los árboles utilizados para este análisis se presentan y resumen en la Tabla 1.

\section{Modelos para determinar el ancho de la corona}

En la presente investigación fueron utilizadas funciones lineales y no lineales, utilizando las variables como ancho de corona, diámetro a la altura del pecho y largo de corona para probar el modelo que más se ajuste para la descripción de ancho de corona del Laurel bajo de sistemas agroforestales, en el Bosque Protector Sumaco. Las funciones que fueron empleadas en esta investigación son presentadas en la Tabla 2.

\section{Modelos para determinar la base de corona}

Para la descripción de la base de corona (Ka) del Laurel bajo sistemas agroforestales, en el Bosque Protector Sumaco, se utilizaron los modelo propuesto por: el Programa Silva [23], el modelo empleado por el Programa de Modelación de Crecimiento FOSTER [24], la versión modificada del Programa de Modelación de Crecimiento FOSTER [9], el modelo planteado por Durski [25], el modelo desarrollado por Nagel [26]. y el modelo propuesto por Monserud [27], cuyas funciones son presentados en la Tabla 3.

\section{Modelos para determinar el largo de corona}

Para la estimación de este parámetro se desarrolló un modelo no lineal, el cual empleó una serie de variables que permitieron explicar el largo de corona en sistemas agroforestales dentro del Bosque Protector Sumaco.

\section{Evaluación y validación de los modelos}

Todas las estimaciones de los parámetros empíricos de los modelos propuestos fueron evaluadas mediante el programa STATISTICA [28], de acuerdo al cuadrado medio del error, el cual fue el menor posible, que el número de parámetros del modelo propuesto sea lo menor posible (principio de simplicidad), que el error estándar de las estimaciones de los parámetros sea lo más pequeño posible y que los coeficientes estimados no se encuentren altamente correlacionados [29]. Adicionalmente, se realizó una evaluación cuantitativa de modelos de crecimiento que puede incluir una caracterización del error en términos de su magnitud y la distribución de los residuos, probar su distorsión, precisión y un análisis de sensibilidad para identificar aquellos componentes que tienen gran influencia en los predictores [16].

La asunción de un modelo de regresión es que el error $\varepsilon$, por ejemplo en $Y=\alpha+\beta X+\varepsilon$ es aditivo, independiente y distribuido normalmente, con un promedio y una constante variación no conocida. Estas características representan condiciones ideales, que normalmente no son observadas, cuando se realizan modelos.

\begin{tabular}{|c|c|c|c|}
\hline & \multicolumn{3}{|c|}{$\begin{array}{c}\text { Datos del Modelo de Ancho } \\
\text { de la Corona } \\
(\mathrm{N}=207)\end{array}$} \\
\hline & Mín. & Máx. & Prom. \\
\hline DAP & 10.0 & 37.5 & 23.9 \\
\hline Altura Total & 7.9 & 23.6 & 17.7 \\
\hline Ancho de Corona & 1.3 & 5.6 & 3.3 \\
\hline Base de Corona & 5.6 & 18.1 & 11.5 \\
\hline
\end{tabular}

Tabla 1: Datos generales de los árboles utilizados para este análisis de corona $(\mathrm{N}=$ número de árboles; $\mathrm{DAP}=$ diámetro a la altura del pecho; Min. = mínimo; Max. = máximo; Prom. = media $)$, Bosque Protector Sumaco, provincia de Napo. 


\begin{tabular}{cl}
\hline Nominación & \multicolumn{1}{c}{ Función } \\
\hline $\mathrm{AC}_{1}$ & $A C=a+b * d a p$ \\
$\mathrm{AC}_{2}$ & $A C=a+b * d a p+c * h$ \\
$\mathrm{AC}_{3}$ & $A C=a+b * d a p+c * h+d * L C$ \\
$\mathrm{AC}_{4}$ & $A C=a * d a p^{b}$ \\
$\mathrm{AC}_{5}$ & $A C=a * d a p^{b} * h^{c}$ \\
$\mathrm{AC}_{6}$ & $A C=a * d a p^{b} * h^{c} * L C^{d}$ \\
$\mathrm{AC}_{7}$ & $\ln (A C)=a+b * \ln ($ dap $)$ \\
\hline AC $=$ Ancho de corona en m; dap = Diámetro a la \\
altura del pecho en cm; $h=$ Altura total en m; LC \\
largo de la corona; $a, b, c$, $d=$ parámetros del \\
modelo; ln = logaritmo natural \\
\hline
\end{tabular}

Tabla 2: Funciones para determinar en ancho de la corona adaptada para Laurel, en sistemas agroforestales, Bosque Protector Sumaco, provincia de Napo.

\begin{tabular}{cc}
\hline Nominación & \multicolumn{1}{c}{ Función } \\
\hline Ka-Silva & $K a=h *\left[1-e^{(a+b * h / d a p+c * d a p)}\right]$ \\
Ka-Forest & $K a=h * e^{a * h^{b} *(d a p+c)^{d}}$ \\
Ka-Forest & \\
modificado & $K a=h * e^{a * h^{b} * d a p^{c}}$ \\
Ka-Ďurský & $K a=h *\left(a+\left[e^{b *\left(\frac{h_{i}}{d a p_{i}}-0,4\right)}\right]\right)$ \\
Ka- Nagel & $K a=h *\left[1-e^{-\left(a+b * \frac{h_{i}}{d_{i}}\right)^{2}}\right]$ \\
Ka- Monserud & $K a=h * e^{\left(b_{1} * h^{\left.b_{2} * d\right)}\right.}$ \\
\hline $\begin{array}{l}\text { Ka }=\text { base de corona en } m ; h=\text { Altura total en } m ; \\
\text { dap }=\text { Diámetro a la altura del pecho en cm; } \\
a, b, c \text {, d = parámetros del modelo }\end{array}$ \\
\hline
\end{tabular}

Tabla 3: Funciones para determinar el ancho de la corona adaptada para Laurel, en sistemas agroforestales, Bosque Protector Sumaco, provincia de Napo.

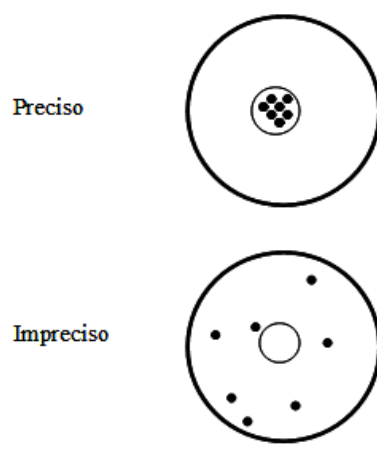

No distorsionado

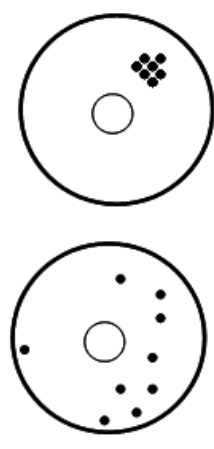

Distorsionado
Figura 4: Ilustración del concepto de precisión y distorsión. Una predicción precisa y no distorsionada es exacta.

El error esta caracterizado por su magnitud y la distribución de sus residuos. Estos pueden ser evaluados mediante la comparación del modelo propuesto y el conjunto de observaciones. Conceptos importantes para la evaluación de modelos es la distorsión y precisión, los cuales determinan la exactitud de la predicción (Figura 4).

En esta contribución fueron esgrimidos criterios cuantitativos para evaluar, comparar y validación de los modelos desarrollados, como medida para comparar los datos observados y predichos mediante los modelos propuestos por [30]. Los criterios para la evaluación de los modelos fueron de acuerdo a la ecuación 1 (Distorsión) y 2 (Precisión):

$$
D=\frac{\sum_{i=1}^{n}\left(y_{i}-\hat{y}_{i}\right)}{n}
$$

El valor ideal de la dipersión es 0 .

$$
R M S E=\sqrt{\sum_{i=1}^{n} \frac{\left(y_{i}-\hat{y}_{i}\right)^{2}}{n-p}}
$$

El valor ideal de Cuadrado Medio del Error (RMSE) es 0. Donde: $d_{i}=y_{i}=$ Datos observados; $\hat{y}_{i}=$ Datos calculados (Predichos); $n=$ Total de observaciones; $p=$ Número de parámetros empleados en los modelos, Cuadrado Medio del Error.

\section{Resultados y Discusión}

\section{Ancho de corona}

Los coeficientes de los parámetros, coeficiente de determinación, distorsión y el cuadrado medio del error (RMSE) son exhibidos en la Tabla 4.

El Laurel como otra especie de crecimiento rápido, crece mejor, cuando tiene amplio espacio para crecer y su corona se puede desplazar, especialmente en edades tempranas y cuando la mayor altura ha sido alcanzada [31]. De acuerdo a los valores de distorsión de 0.0375 $\mathrm{m}$., y de precisión de $0.4776 \mathrm{~m}$. (RMSE) observados para $\mathrm{AC}_{1}$, se acercaron más a los valores ideales de cero y se puede reconocer también que, al añadir variables como la altura y el largo de la corona, no lleva a un mejoramiento significativo de esta estimación de este parámetro. Tanto [32] como [33] señalaron que, una simple relación lineal entre diámetro y ancho de corona, es la mejor función para determinar este parámetro para especies de cuatro estaciones. No obstante, investigaciones realizadas por [34] quien, incluyó altura total y largo de corona, mejorando la estimación del ancho de corona en comparación con los modelos que incluyen únicamente al diámetro a la altura del pecho como variable independiente. Este hecho se ajustó para el Laurel, de ahí que, la relación lineal Ancho de Corona $=0.33+$ $0.12 * D A P$ es la mejor opción para establecer el ancho de la corona para Laurel dentro de sistemas agroforestales.

\section{Base de corona}

En cuanto a los modelos que permiten estimar la longitud de la copa, la mayoría se pueden englobar en dos grandes grupos: En el primero se incluyen todos los modelos que permiten estimar la altura a la que comienza la copa [7] y este fue el método empleado en esta investigación. El segundo grupo incluye los modelos que estiman el ratio de copa, es decir, el cociente entre la 


\begin{tabular}{cccccccc}
\hline Ecuación & \multicolumn{9}{c}{ Coeficientes } & & $\mathbf{r}^{2}$ & Distorsión (m) & RMSE (m) \\
\hline & $\mathrm{a}$ & $\mathrm{b}$ & $\mathrm{c}$ & $\mathrm{d}$ & & & \\
\hline $\mathrm{AC}_{1}$ & 0.3327 & 0.1215 & & & 0.6685 & 0.0375 & 0.4776 \\
$\mathrm{AC}_{2}$ & 0.2700 & 0.1149 & 0.0125 & & 0.6686 & 0.0386 & 0.5667 \\
$\mathrm{AC}_{3}$ & 0.2719 & 0.1197 & -0.0189 & 0.0707 & 0.6766 & 0.0387 & 0.6797 \\
$\mathrm{AC}_{4}$ & 0.1917 & 0.8914 & & & 0.6685 & 0.0392 & 0.6047 \\
$\mathrm{AC}_{5}$ & 0.1819 & 0.8425 & 0.0720 & & 0.6686 & 0.0395 & 0.6418 \\
$\mathrm{AC}_{6}$ & 0.2072 & 0.8660 & -0.0844 & 0.1360 & 0.6782 & 0.0485 & 0.5087 \\
$\mathrm{AC}_{7}$ & -1.2476 & 0.7592 & & & 0.5994 & 0.0555 & 0.8106 \\
\hline
\end{tabular}

Tabla 4: Valores calculados, coeficiente de determinación y el cuadrado medio del error (RMSE) para los diversos modelos de ancho de corona, Bosque Protector Sumaco, provincia de Napo.

altura de la copa y la altura total del árbol. A este grupo pertenecen, entre otros, los modelos de Hassenauer [35] o Sterba [36]. El modelo de copa desarrollado por Sterba [37] para pícea tiene la siguiente expresión:

$$
C R=\frac{1}{1+\exp \left(a_{0}+\sum_{i} a_{i} \cdot E s t_{i}+\sum_{j} b_{j} \cdot \operatorname{Dim}_{j}+\sum_{k} c_{k} \cdot \text { Com }_{k}\right)}
$$

Donde $C R$ es el ratio de copa, $E s t_{i}$ es un vector de variables de la estación, $\operatorname{Dim}_{j}$ es un vector de las dimensiones del árbol (diámetro, relación $h / d$ y altura del árbol) y $\mathrm{Com}_{k}$ es un vector que define la competencia con índices como el denominado Crown Competition Factor de Ktajicek [38].

En la Tabla 5, se presenta los coeficientes calculados para este parámetro, el coeficiente de determinación, distorsión y el cuadrado medio del error. De los diferentes modelos probados para el Laurel, se puede destacar el desarrollado por [25], que es el que mayor r (0.8453) presentó, los valores de distorsión del mencionado modelo se acercan a los valores ideales de $0(0.0083)$ y el cuadrado medio del error fue de 1.49 metros.

En la Figura 5, se presenta el análisis de residuos, los valores observados frente a los valores predichos por el modelo [25], en ningún caso se observó una distorsión significativa estadística de los residuos. Este modelo puedo ser empleado para predecir la base de la corona del Laurel bajo un manejo agroforestal en el Bosque Protector Sumaco.

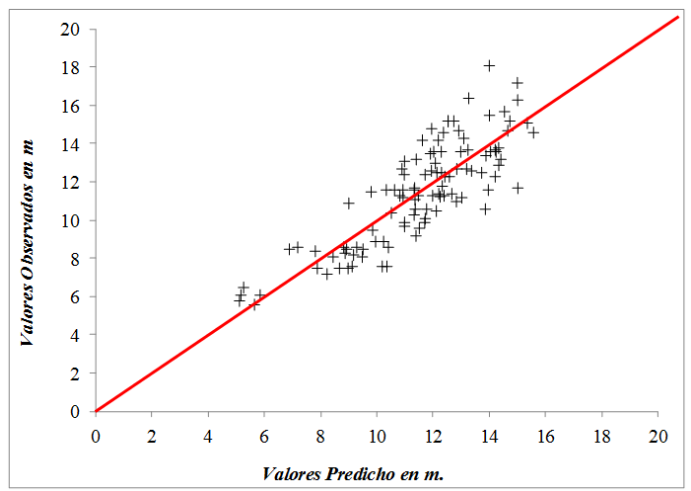

Figura 5: Valores observados vs valores predichos para el modelo de Durský (2000). Así como la dispersión de residuos del mencionado modelo, Bosque Protector Sumaco, provincia de Napo.
$L C=H * e^{(-a * g+b *(H / D A P)+c * \log (G / H)+d * \log (D A P))}$

Donde $L C=$ Largo de la corona; $H=$ Altura total en $\mathrm{m} ; G=$ Área basal en $\mathrm{cm}^{2} ; H / D A P=$ Relación altura diámetro; $D A P=$ Diámetro a la altura del pecho en $\mathrm{cm}$ y $a, b, c, d=$ Parámetros empíricos del modelo.

Los valores para el parámetro $a=1.630759 ; b=0.421923$; $c=0.063353$ y $d=-0.218853$, se obtuvo un coeficiente de determinación r de 0,71 . Esto significa que el $71 \%$ de la variación observada en el Laurel para este parámetro puede ser explicada mediante el modelo propuesto. La distribución normal esperada de los residuos son presentados en la Figura 6. La determinación de este parámetro permitirá determinar los niveles de competencia de un rodal agroforestal por un largo tiempo [39]. Permite también establecer la vitalidad del componente arbóreo de los sistemas agroforestales [40] y da información sobre la potencial reacción de una intensidad de raleo [41].

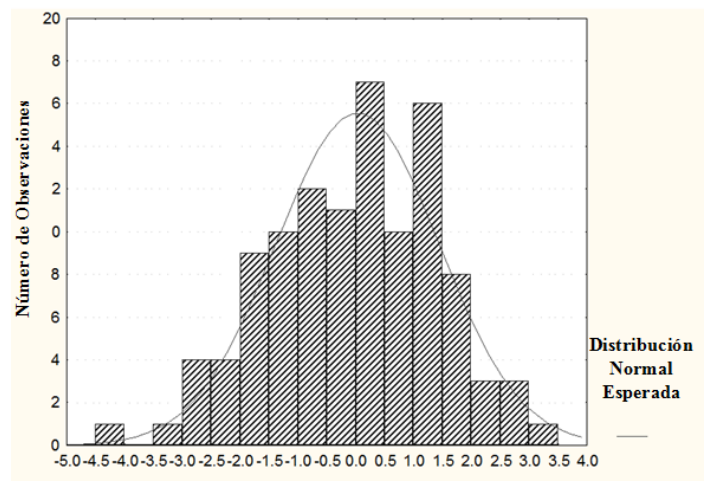

Figura 6: Distribución de los residuos en confrontación con el número de observaciones realizados y la distribución normal esperada para el modelo de largo de corona, Bosque Protector Sumaco, provincia de Napo.

\section{Proyección de corona}

Los sistemas agro-silvopastoriles interactúan entre los diferentes componentes en términos de la ocupación de espacio (estratificación vertical y horizontal, sobre y debajo de la tierra) y asignación de recursos (luz, agua, nutrientes) [42]. Dos diferentes interacciones podrían ser definidas: 


\begin{tabular}{ccccccc}
\hline Ecuación & \multicolumn{3}{c}{ Coeficientes } & $\mathbf{r}^{2}$ & Distorsión (m) & RMSE (m) \\
\hline & $\mathrm{a}$ & $\mathrm{b}$ & $\mathrm{c}$ & & & \\
\hline Ka-Silva & -0.4472 & 0.4169 & -0.3878 & 0.8374 & 0.0384 & 1.5022 \\
Ka-Forest & 1.1739 & -0.2040 & & 0.8378 & 0.0478 & 1.4967 \\
Ka-Forest modificado & 1.5751 & -0.6081 & -0.0030 & 0.8382 & 0.0429 & 1.4984 \\
Ka-Durský (2000) & -0.3370 & 9.4999 & 11.5614 & 0.8453 & 0.0083 & 1.4951 \\
Ka- Nagel (1999) & 1.1732 & -0.2020 & & 0.8433 & 0.0106 & 1.4993 \\
Ka- Monserud (1975) & -0.8014 & -1.3189 & & 0.8149 & -0.0199 & 1.6023 \\
\hline
\end{tabular}

Tabla 5: Valores calculados, coeficiente de determinación, distorsión y el cuadrado medio del error (RMSE) para los diversos modelos de ancho de corona, Bosque Protector Sumaco, provincia de Napo.

- Interacción Directa: componentes abióticos con otro componente biótico (árbol con otros cultivos)

- Interacción Indirecta: un componente biótico modifica a un componente abiótico usado por otro componente biótico (reducción de la luz resultante de la sombra de un árbol).

Este parámetro de proyección de corona podría ser empleado para el desarrollo de modelos que involucren una serie de componentes para comprobar la productividad de los sistemas agro-silvopastoriles. Los modelos desarrollados dentro de esta investigación pueden ser empleado para establecer y calcular otro tipo de atributos como por ejemplo la proyección en superficie de la corona al suelo (Ecuación 5). Esta proyección en unidad de área (PC) se deduce del largo de la corona (LC= $\left.\mathrm{RC}^{*} \mathrm{H}\right)$ y donde el radio de la corona se obtiene del ancho de la corona $(\mathrm{RC}=\mathrm{LC} / 2)$ de acuerdo al modelo desarrollado por [43].

$$
P C=\frac{\pi * R C}{6 * L C^{2}}\left[\left(4 * L C^{2}+R C^{2}\right)^{\frac{3}{2}}-R C^{3}\right]
$$

Donde $P C=$ Área de proyección de la corona $\left(\mathrm{m}^{2}\right) ; R C$ $=$ Radio de la corona $(\mathrm{m}) ; L C=$ Largo de la corona $(\mathrm{m})$ y $\pi=3.1416$.

\section{Conclusiones}

Los resultados de esta investigación sobre la descripción de corona del laurel, demuestran una cierta variación de acuerdo a la edad de los sistemas agroforestales investigados. Para la descripción de los atributos de la corona se utilizaron las siguientes funciones: $y=\frac{1}{1+e^{x}}$, $y=e^{-\sqrt{x}}, y=e^{-x}$.

El crecimiento del árbol entre otros parámetros forestales como la base y ancho de corona pueden ser determinados mediante funciones matemáticas. Estos parámetros pueden también ser deducidos para describir este componente forestal de los sistemas agroforestales, cuando se dispone de parámetros que normalmente son tomados para la caracterización de masas forestales. Esto es el diámetro a la altura del pecho y estimación de altura, que normalmente son estimados para modelos de crecimiento. Estas valoraciones pueden complementarse a través de la introducción de un modelo de la corona.
Es necesario realizar nuevas investigaciones para determinar la influencia de las diferentes edades de los árboles, densidades, alturas y grados de competencia entre coronas dentro de sistemas agroforestales para determinar la estabilidad y vulnerabilidad de estos sistemas ante la presencia de vientos alisos huracanados en la Región Amazónica Ecuatoriana.

\section{Referencias}

[1] Ministerio del Ambiente del Ecuador, M. 2005. "Evaluación y actualización de las estrategias de desarrollo forestal sustentable del Ecuador, estrategia para el desarrollo forestal sustentable del Ecuador (2005)". Ministerio del Ambiente del Ecuador (MAE). Quito, Ecuador.

[2] MAGAP 2008. "Plan de reactivación agropecuario 2007-2011”. Ministerio de Agricultura, Acuacultura y Pesca del Ecuador: Quito, Ecuador.

[3] Cañadas, A. 2005. "Providing information about natural resources as a base to support the decentralization of the forest sector in canton Loreto". Research Centre of Forest Ecology, University Göttingen: Niedersachsen, Alemania.

[4] Cañadas, A.; Arce, L.; Molina, C. 2010. "Growth, yield and performance of teak in silvopastoral system in the lowland western region, ecuador". World Food System A Contribution from Europe: Tropentag. Zurich.

[5] Cañadas, A.; Roca, A. 2011. "Can the reforestation projects stop the extraction of wood from the protected forest chongón-colonche?". Tropentag, Bonn University Development on the Margin.

[6] Van Laar, A.; Akca, A. 1997. "Forest mesuration". Cuvilier Verlag: Göttingen, Deutschland.

[7] Gadow, K.; Hui, G. 1999. "Modeling forest development". Kluwer Academic Publisher.

[8] Gadow, K. 2004. "Forsteinrichtung. steuerung und analyse der waldentwicklung". Beilage zur Vorlesung, Fakultät für Forstwissenschaften und Waldökologie, Universität Göttingen. Göttingen, Deutschland.

[9] Hasenauer, H. 1994. "Ein einzelbaumwachstumssimulator für ungleichaltrige fichten-kiefern- und buchen- fichtenbestände". Dissertation, Universität für Bodenkultur, Wien.

[10] Raven, P.; Evert, R.; and Eichhorn, S. 1987. "Biology of plants". Worth Publishers Inc.: New York, NY.

[11] Mitscherlich, G. 1970. "Wald, wachstum und umwelt". Form und Wachstum von Baum und Bestand. 
[12] Leuschner, C. 2002. "Lebensraum baumkrone Û schatzkiste der biodoversität". Georgia August, Wissenschaftsmagazin der Geor-August-Universität. Göttingen, Deutschland.

[13] Korol, M.; Gadow, K. 2002. "Zur quantitativen beschreibung von fichtenkronen im gebiet der ukrainischen karpaten". Unveröff, Manuskript, Inst. f. Waldinventur u. Waldwachstum, Univ. Göttingen, Deutschland.

[14] Korol, M.; Gadow, K. 2003. "Ein einheitsschaftmodell für die baumart fichte". Forstwissenschaftliches Centralblatt, 122(3): 175-182.

[15] Kramer, H. 1988. "Waldwachstumslehre". Verlag Paul Parey. 800.

[16] Gadow, K. 2003. Waldstruktur und Wachstum: Beilage zur Vorlesung im Wintersemester 2003/2004. Universitätsverlag Göttingen: Göttingen, Alemania.

[17] Badoux, E. 1939. "De l'influence de divers modes et degres d"eclaircie dans les hetraies pines. mittschweiz". AFV. 21(1): 59-146.

[18] Ung, C. 1993. "Growth and branchiness of picea mariana (mill.) bsp". Canadian Forest Service Modelling Working Group. 200.

[19] Proyecto Gran Sumaco 1998. "Distribución de lluvias en la estación de chalua yacu". PGS/INEFAN/GTZ. 10.

[20] Bendix, J.; Lauer, W. 1992. "Die niederschlagsjahreszeiten in ecuador und ihre klimadynamiche interpretation". Erkunde. 118-134.

[21] Cañadas, L. 1979. "Identificación de sistemas ecológicos en la región amazónica ecuatoriana”. Seminario sobre Manejo de Sistemas Ecológicos y Alternativas de Producción Agro-Silvo-Pastoril, Limoncocha. 16.

[22] Cañadas, L. 1983. "Mapa bio climático y ecológico del ecuador". Editores Asociados Cia. Ltd.: Quito, Ecuador.

[23] Pretzch, H.; Kahn, M. 1998. "Forschungsvorhaben konzeption und konstruktion von wuchs und prognosemodellen für mischbeständenin bayer". Methodische Grundlagen. 279.

[24] Ek, A. 1974. "Dimensional relationships offorest and open-grown stems in wisconsin". Uni. of Wisconsin, Forestry Tes. 7 .

[25] Ďurský, J. 2000. "Einsatz von waldwachstumssimulatoren für bestand, betrieb und grobregion". Dissertation. 223.

[26] Nagel, J. 1999. "Konzeptionelle Überlegungen zum schrittweisen aufbau eines waldwachstumskundlichen simulationssystems für nordwestdeutschland". Franfurt am Main. 122.

[27] Monserud, R. 1975. "Methodology for simulating wisconsin northern hardwood stand dynamics". Ph.D. Thesis. Univ. of Wisconsin. 156.

[28] Statistica 2004. "User's Guide". STATISTICA Version 5.0 .

[29] Bortz, J. 2005. "Statistik für sozialwissenschaftler". Springeler-Verlag.

[30] Sterba, H.; Korol, M.; Rössler, G. 2001. "Ein ansatz zur evaluierung eines einzelbaumwachstumssimulators für fichtenreinbestände". Forstwissenschaftliches Centralblatt. 406-421.
[31] Somarriba, E.; Valdiviezo, R.; Vásquez, W.; Galloway, G. 2001. "Survival, growth, timber productivity and agro forestry systems". Agroforestry Systems. 111-118.

[32] Zeide, B. 1983. "The mean diameter for stand density index”. Can. J. For. Res. 1023-1024.

[33] Nagel, J. 1997. "Bwin - program for standard analysis and prognosis". User's manual for version 3.0, Nidersächsische Forsliche Versuchtanstalt, Göttingen, Deutschland.

[34] Moere, M. 1981. "Crown width and foliage weight of northern rocky mountain conifers". USDA For. Serv. Res. 283.

[35] Hassenauer, H.; Monserund, A. 1996. "A crown ratio model for austrian forest". Forest Ecology and Management. 49-60.

[36] Sterba, H. 1987. "Estimating potential density from thinnings experiments and invetory data". Forest Science. $1022-1034$

[37] Sterba, H. 1995. "Prognaus - ein abstandsunabhängiger wachstumssimulator für gleichaltrige mischbestände". Deutscher Verband Forstlicher Forschungsanstaltensektion Ertragskunde. 173-183.

[38] Krajicek, J.; Brinkman, K.; and Gingrich, S. 1961. "Crown competition. A measure of density". For. Sci. $35-42$.

[39] Mitscherlich, G. 1978. "Wald, wachstum und unwelt". Frankfurt am Main, Ed. Sauerländer.

[40] Valentine, H.; Ludlow, A.; Furnival, G. 1994. "Modeling crown rise in even-aged stand of sitka spruce and loblolly pin". For. Ecolog. Manag. 189-197.

[41] Dyer, E.; Burkhart, E. 1987. "Compatible crown ratio and hight models". Can. J. For. Res. 572-574.

[42] Anderson, L.; Sinclair, F. 1993. "Ecological interactions in agroforestry systems". Agrofor. Abs. 57-91.

[43] Kramer, H.; Akca, A. 1982. "Leitfaden für dendrometrie und bestandesinventur”. J.D. Sauerländer's Verlag. 150. 